\title{
Effective Teaching of Theory Course Using PBL
}

\author{
S K. Khamuruddeen ${ }^{1}$, Azeem Unnisa ${ }^{2}$ \\ ${ }^{1,2}$ Department of ECE \\ Hyderabad Institute of Technology and Management \\ Hyderabad, India \\ ${ }^{1}$ khd485@gmail.com, ${ }^{2}$ azeemunnisa.ece@ hitam.org
}

\begin{abstract}
Digital image processing is a method to perform arithmetic operations on an image to improve its quality. Digital image processing has many advantages over analog image processing. Now a Day the usage of Digital Image Processing all over the world has increased in different domains. There are many opportunities for a fresher graduate with Digital Image Processing skills. So, to know the different techniques of Digital Image Processing Project Based Learning Methodology is found very useful to the fresher graduates. Project Based Learning Methodology is implemented by using MATLAB Software and applied to this course. This Paper Presents the Procedure to implement a Project Based Learning Methodology to develop various applications in the area of Digital Image Processing. By Using Project Based Learning we can motivate the students more effectively.
\end{abstract}

Key Words: Digital Image Processing, Project Based Learning Methodology, MATLAB.

\footnotetext{
S K. Khamuruddeen ${ }^{1}$

1,2 Department of ECE

Hyderabad Institute of Technology and Management

Hyderabad, India

${ }^{1}$ khd485@gmail.com
}

\section{I.INTRODUCTION}

Image processing mainly classified in to two types, analogue and digital image processing. Analogue image processing can be used for the hard copies like printouts and photographs. Image analysts use various fundamentals of interpretation while using these visual techniques. Digital image processing techniques help in manipulation of the digital images by using computers. The three general Phases that all types of data have to undergo while using digital technique are pre-processing, enhancement, and display, information extraction. Digital image processing is the process of digital images with various techniques like restoration, segmentation, edge detection Median filtering, Image Addition, subtraction, multiplication and Division. Visual information is the most important type of information perceived, processed and interpreted by the human brain. One third of the cortical area of the human brain is dedicated to visual information processing.

Digital image processing, as a computer based technology, carries out automatic processing, manipulation and interpretation of such visual information, and it plays an increasingly important role in many aspects of our daily life, as well as in a wide variety of disciplines and fields in science and technology, with applications such as television, mobiles, aero engineering, photography, robotics, remote sensing, medical ,biological image processing ,automatic character reorganization and industrial applications.

By Using Project Based Learning Methodology interest in learning to effectively improve the students' practical and innovative ability, and cultivating innovative practice ability in the teaching process. Digital image processing concepts can easily be understood if we teach using visual, experimental methods.

\section{II.DESIGN OF PROJECT BASED LEARNING METHODOLOGY}


Project Based Learning Methodology is applied to Electronics and communication engineering at for IVth Year students at Hyderabad Institute of Technology and Management (HITAM), Jawaharlal Nehru Technological University, Hyderabad. Digital image processing course for students in programming and have some basic knowledge of mathematics require high starting point, it is difficult, and it is based on the theory of many engineering applications, it is taught in the Course process to stimulate students' creative thinking, improve students' practical ability . With the rapid development of image processing technology, the teaching content of the course is also constantly updated.

There are Many Opportunities to the students to do their mini and major projects on digital image processing by using MAT Lab soft Ware. I gave following topics like Histogram Equalization, Median filtering, Image Addition, subtraction, multiplication and Division as a mini projects and it is very useful to the students for active learning.

By the end of this course, students will be able to the basic theory and algorithms that are widely used in digital image processing

\section{METHODOLOGY FOLLOWED FOR PROJECT BASED LEARNING}

For this Course We are having 5 hours per Week in that we use 4 hours for Tutorial Classes and 1 hour per Practical Lab out of those Tutorial Classes. After practical lab students are very confident about all the topics covered in previous tutorial classes.

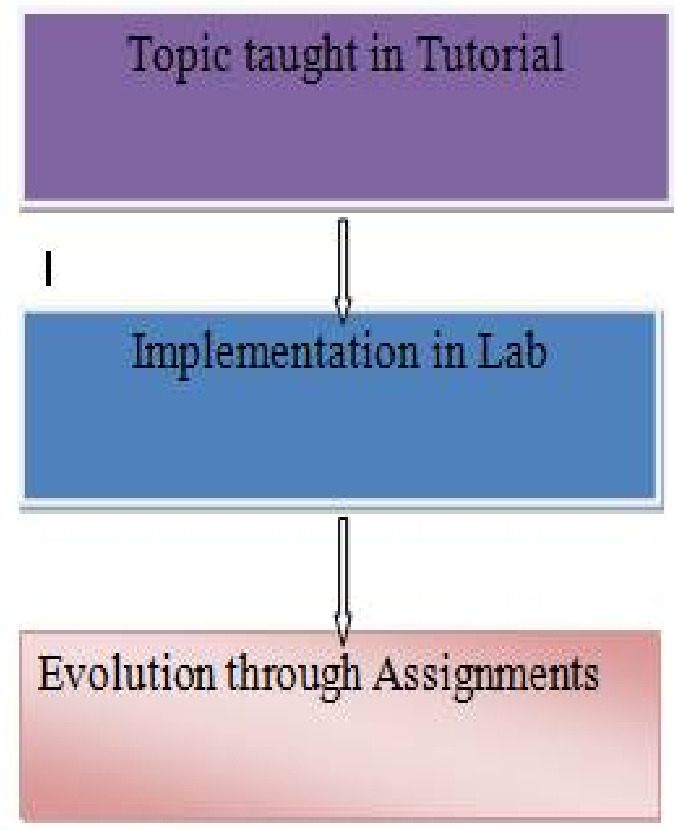

Fig-2: Implementation of PBL

\section{A. Histogram Equalization}

Histogram Equalization usually increases the global contrast of many images, especially when the usable data of the image is represented by close contrast values. Through this adjustment, the intensities can be better distributed on the histogram. This allows for areas of lower local contrast to gain a higher contrast. Histogram equalization accomplishes this by effectively spreading out the most frequent intensity values.

The method is useful in images with backgrounds and foregrounds that are both bright or both dark. In particular, the method can lead to better views of bone structure in $\mathrm{x}$ ray images, and to better detail in photographs that are over or under-exposed. A key advantage of the method is that it is a fairly straightforward technique and an invertible operator. So in theory, if the histogram equalization function is known, then the original histogram can be recovered. 


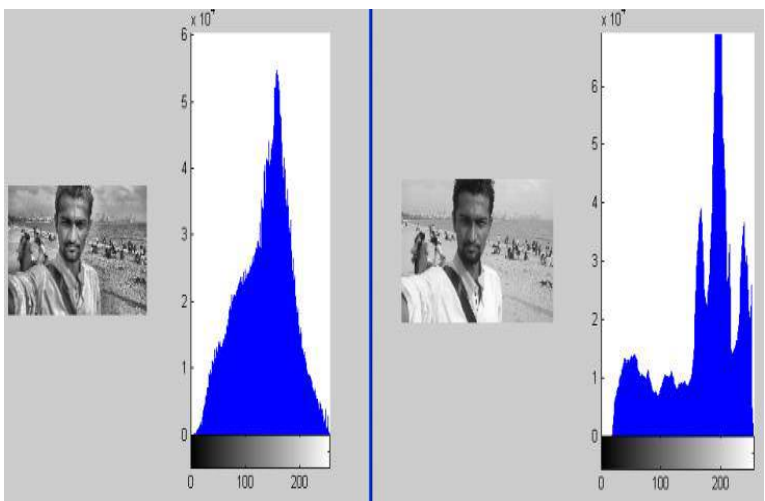

\section{Fig-3: Histogram Equalization}

\section{B. Median filtering}

The main idea of the median filter is to run through the signal entry by entry, replacing each entry with the median of neighboring entries. The pattern of neighbors is called the "window", which slides, entry by entry, over the entire signal. For 1D signal, the most obvious window is just the first few preceding and following entries, whereas for 2D (or higher-dimensional) signals such as images, more complex window patterns are possible (such as "box" or "cross" patterns). Note that if the window has an odd number of entries, then the median is simple to define: it is just the middle value after all the entries in the window are sorted numerically. For an even number of entries, there is more than one possible median.

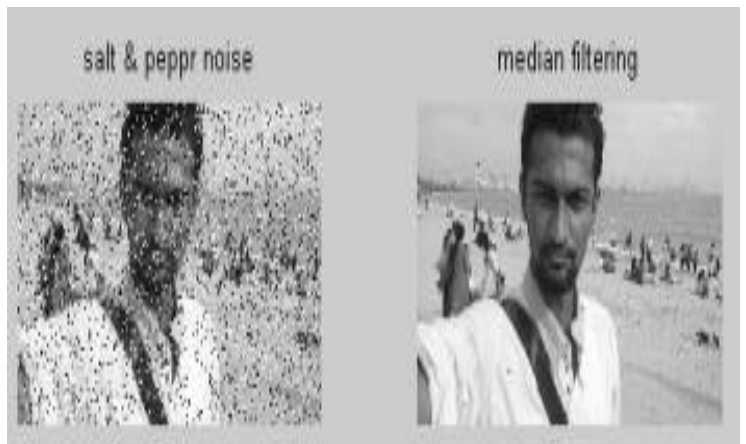

\section{Fig-4: Median filtering}

C. Image addition

Here students takes as input two identically sized images and produces as output a third image of the same size as the first two, in which each pixel value is the sum of the values of the corresponding pixel from each of the two input images. More sophisticated versions allow more than two images to be combined with a single operation. A common variant of the operator simply allows a specified constant to be added to every pixel.

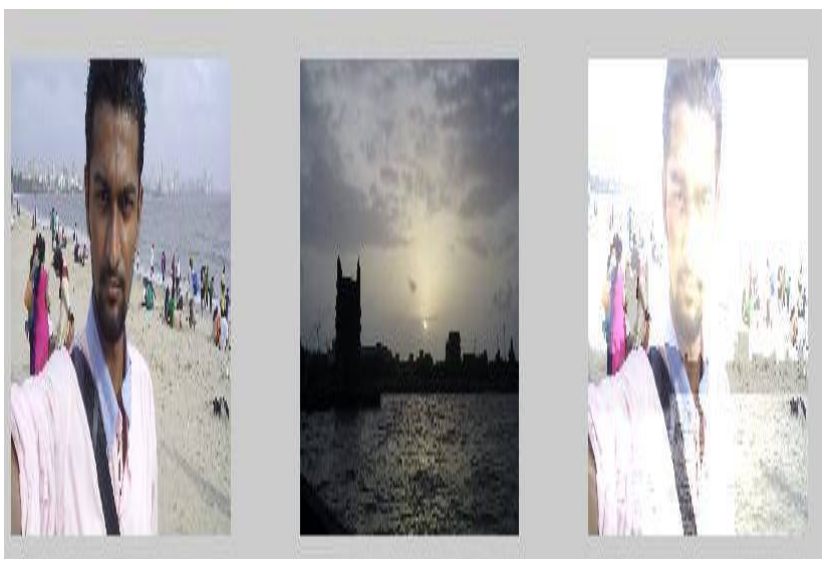

Fig-5: Image addition

\section{Image subtraction}

Here student's takes two images as input and produces as output a third image whose pixel values are simply those of the first image minus the corresponding pixel values from the second image. It is also often possible to just use a single image as input and subtract a constant value from all the pixels. Some versions of the operator will just output the absolute difference between pixel values, rather than the straightforward signed output.

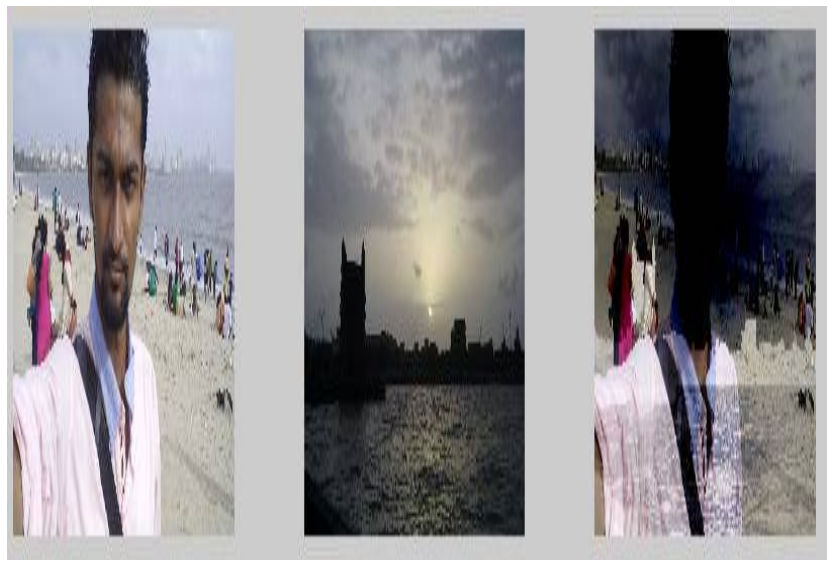

\section{Fig-6: Image subtraction}

\section{E. Image Multiplication}

Like other image arithmetic operators, multiplication comes in two main forms. The first form takes two input images and produces an output image in which the pixel values are just those of the first image, multiplied by the values of the corresponding values in the second image. The second form takes a single input image and produces output in which each pixel value is multiplied by a specified constant. 


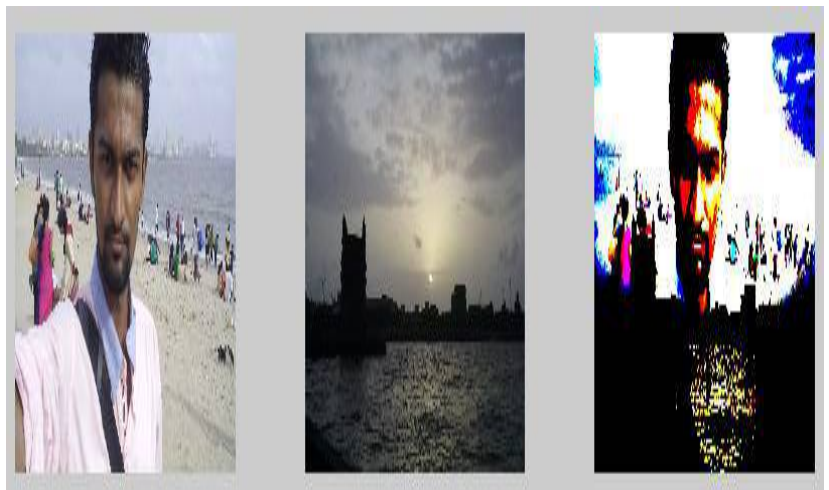

Fig-7: Image Multiplication

\section{F. Image Division}

The image division operator normally takes two images as input and produces a third whose pixel values are just the pixel values of the first image divided by the corresponding pixel values of the second image. Many implementations can also be used with just a single input image, in which case every pixel value in that image is divided by a specified constant.

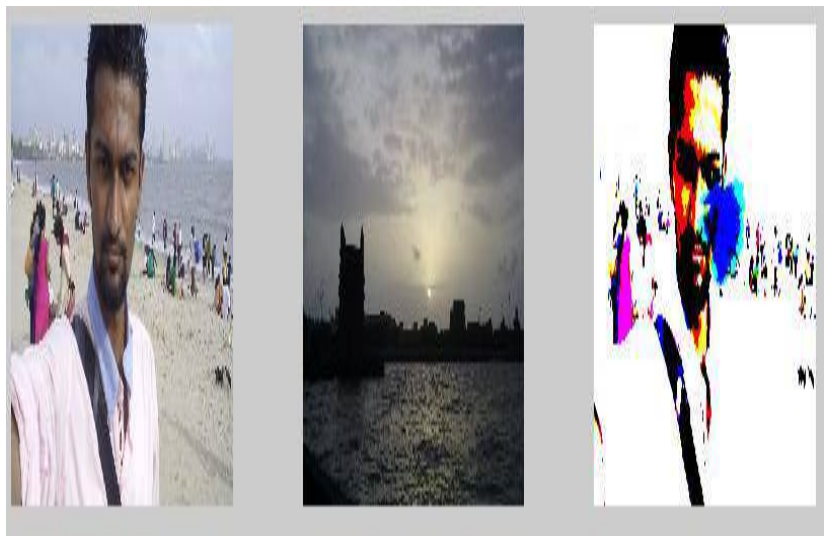

Fig-8: Image Division

\section{Conclusion}

This Paper describes a Project Based Learning Methodology developed at the Electronics and communication engineering at for IVth Year students at Hyderabad Institute of Technology and Management (HITAM), Jawaharlal Nehru Technological University, Hyderabad, to teach a Digital image processing course. This Methodology is very useful to the students to understand the concepts easily and actively. After using this method the strength of the class also increased. After this course with the Project Based Learning Methodology students are able to publish their mini/major projects in national/ international journals. Project Based
Learning Methodology does provide a more challenging, motivating and enjoyable approach to education. These processes and learning skills help students become more competent in information-seeking skills than traditional students.

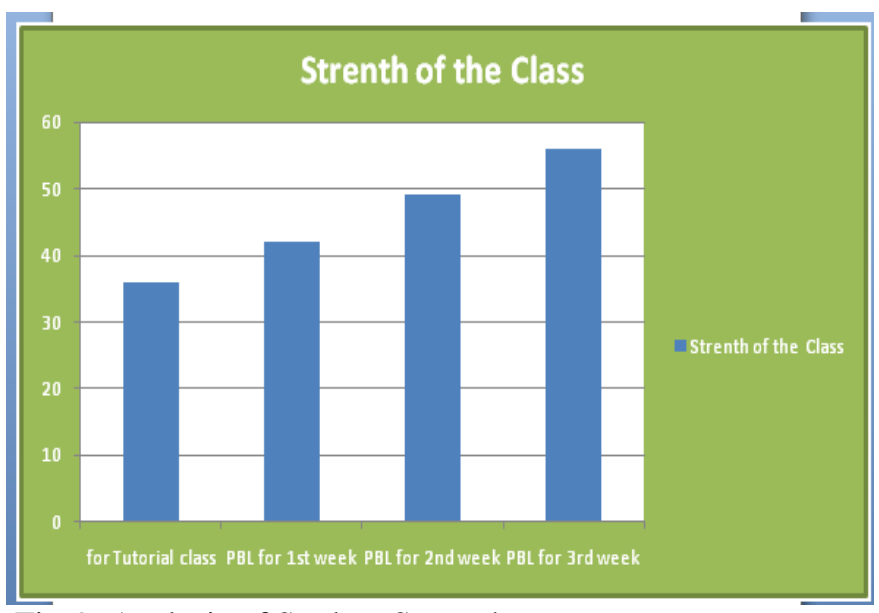

Fig-9: Analysis of Student Strength

\section{Acknowledgement}

We would like thank chairman, Principal, Director and HOD (ECE) of Hyderabad Institute of Technology and Management (HITAM), Hyderabad for their encouragement to publish this Paper.

\section{References}

1. Gonzalez, R.C. and R.E. Woods, Digital Image Processing.

2. Goodman, J.W., Introduction to Fourier Optics. McGrawHill Physical and Quantum Electronics Series

3. Heijmans, H.J.A.M., Morphological Image Operators. Advances in Electronics and Electron Physics. 1994, Boston: Academic Press.

4. "Fundamentals of Digital Image Processing" by Anil K. Jain

5. Young, I.T., Sampling density and quantitative microscopy. Analytical and Quantitative Cytology and Histology, 1988.

6. Kulpa, Z., Area and perimeter measurement of blobs in discrete binary pictures. Computer Vision, Graphics and Image Processing, 1977.

7. Vossepoel, A.M. and A.W.M. Smolders, Vector code probabilities and metrication error in the representation of 
Journal of Engineering Education Transformations, Special Issue, elSSN 2394-1707

straight lines of finite length. Computer Graphics and Image Processing, 1982.

8. Photometric Ltd., Signal Processing and Noise, in Series 200 CCD Cameras Manual. 1990: Tucson, Arizona.

9. Huang, T.S., G.J. Yang, and G.Y. Tang, A Fast TwoDimensional Median Filtering Algorithm. IEEE Transactions on Acoustics, Speech, and Signal Processing, 1979. 